\title{
Simulation of High Voltage Direct Current Filters
}

\author{
Calum J. Mackinnon \\ Calum.Mackinnon@Strath.ac.uk \\ Brian G. Stewart \\ Brian.Stewart.100@Strath.ac.uk \\ Department of Electronic and Electrical Engineering \\ University of Strathclyde \\ Glasgow, Scotland
}

\begin{abstract}
Electrical transmission networks are becoming increasingly interconnected, on continental scales, toward secure and cost-effective supplies of electrical energy met by growing adoption of low-carbon generation. High voltage direct current transmission links employ power electronics to enable this interconnection, of which conventional designs require highfrequency distortion to be filtered by dedicated assets including resistors, reactors, and capacitor banks. These assets are tuned to select for characteristic harmonic frequencies, but operating conditions (firing angle and commutation overlap) also influence high frequency signal composition. This paper presents simulations of these filtering assets to illustrate high frequency signal attenuation, considering the effects of varied operating conditions, filter bank switching, and component degradation.
\end{abstract}

Keywords-harmonics, HVDC, filtering

\section{INTRODUCTION}

High voltage direct current (HVDC) systems are becoming increasingly prevalent within power transmission systems as they offer very high capacity connections; the opportunity to connect asynchronous or otherwise independent networks; and to connect over large distances with particular relevance to connecting subsea, thereby enabling connection of offshore wind at scale. As power electronic technologies, such systems additionally allow power flows to be controlled which can provide further benefits to existing alternating current (AC) network infrastructure, as seen in back-to-back configurations. Their comparative size (fewer conductors and greater use of a conductor cross-section without having a skin effect) also allows existing $\mathrm{AC}$ connections to be restrung for direct current (DC) toward hybrid grid applications [1], and HVDC technologies can even be combined toward multi-terminal direct current (MTDC) networks. The increasing prevalence of HVDC is in part the reason for a recently proposed CIGRE working group with a focus on power system stability despite increasing expected harmonics and system resonance effects [2]. Different topologies of HVDC each provide respective benefits and drawbacks. Conventional HVDC links use thyristor-based valves to maintain near constant DC current, but rely on $\mathrm{AC}$ waveforms to return through a zero-crossing to commutate. Thyristors consist of 'pnpn' semiconductor layers, and are fired by applying a gate voltage. As semiconductor technologies developed, the advent of water-cooled insulated gate bipolar transistors (IGBTs) enabled voltage source converter (VSC) technologies. Since IGBTs can both fire and block according to a control signal, VSC topologies allow greater controllability and consume less reactive power relative to conventional designs, so need less filtering and can connect to passive networks, in turn making them useful in renewable connections and blackstart scenarios. To focus on filtering arrangements, only conventional current source converter (CSC) - otherwise known as natural line commutated converter (LCC) - topologies are considered in this article. LCC systems generate harmonics, so need large converter station footprints for the associated filters, and are best suited to strong $\mathrm{AC}$ networks.

This paper attempts to highlight characteristic harmonics and residual harmonics which remain in a simulated system despite filtering. Section II provides context before Section III explores typical harmonic profiles associated with HVDC systems. Typical filtering arrangements are then discussed in Section IV, which illustrates how filters selectively eliminate certain frequencies and how changes to asset health can affect filtering. Section $V$ then presents a methodology from which results are obtained in Section VI, before discussion and conclusions are drawn in Section VII and in Section VIII respectively.

\section{BACKGROUND}

Conversion from $\mathrm{AC}$ to $\mathrm{DC}$ takes place where a six-valve bridge is switched to pass only positive parts of an alternating current waveform onto one DC terminal, and only negative parts onto the other. As vertebrae allow a straight spine to bear load, a collection of many synchronised switching elements can bear an evenly distributed voltage. The angle at which a switch conducts relative to the voltage waveform is known as the firing angle $(\alpha)$ and the inevitable delay in current reaching its maximum value due to $\mathrm{AC}$-network inductance is known as a commutation overlap angle $(\gamma)$ [3]. These parameters each respectively relate to loading and voltage on the DC link [4]. The nature of AC-to-DC switching produces harmonics characteristic of the switching process and related to operating angles $\alpha$ and $\gamma$, to which sets of AC filters at either end of a conventional HVDC link are specifically tuned to attenuate. Harmonics degrade the quality of a supply and must be kept within defined limits: they introduce network inefficiencies and accelerate asset deterioration. A Simulink model simulates an LCC $1 \mathrm{GW}$ 12-pulse bipole connection between asynchronous $\mathrm{AC}$ networks at different frequencies, with associated control and interfacing components. It can be accessed through the 'power hvdc12pulse' Matlab command and is shown in Figure 1 [5]. This Simulink model forms the basis of the following simulation studies. 


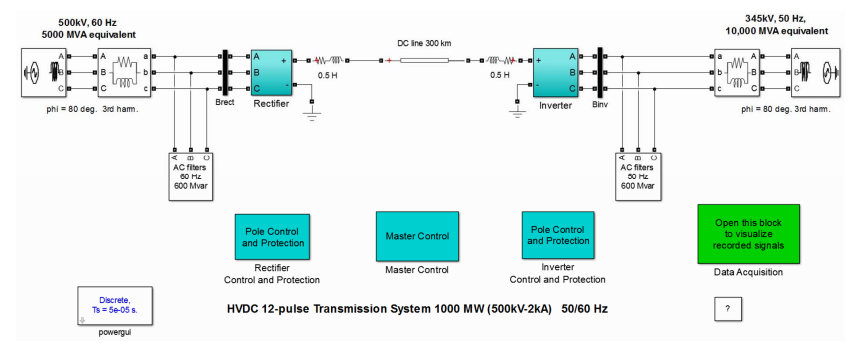

Figure 1: An HVDC transmission link simulation block diagram [5]

Rather than specify parameters for a particular system, those specified in the model are used as the basis of this study, with the following alterations: the model is simplified such that no faults occur, and such that each simulation ends without ramping down in order to capture steady-state behaviour as each simulation terminates. AC voltage source supplies are conveyed through line impedances to pass AC filtering assets prior to reaching the converter ('rectifier' and 'inverter') subsystems, each of which contains a 3-winding converter transformer and two 6-pulse converter bridges. DC line reactors smooth out current transitions, thereby ensuring a smooth line current to reduce inefficiencies on the DC connection. A simulation solver (the 'powergui' block), control and protection subsystems, and a set of preconfigured measurement scopes are also present. The model is augmented by the inclusion of voltage and current measurements on $\mathrm{AC}$ interfaces of the rectifier and inverter blocks and at the $\mathrm{AC}$ filter block terminals; it already includes buses as voltage and current measurement points, but by adding specific voltage and current measurement nodes at these locations, not only can these signals be saved to the workspace for each simulation set with different parameters, but this also allows filter currents to be captured directly. This setup is the basis for a preliminary investigation of converter harmonics and the filtering assets designed to eliminate them.

\section{CONVERTER HARMONICS}

Conventional power electronic converters unavoidably generate characteristic harmonics through normal operation. The character of these harmonics is determined by transformer winding arrangements, the power factor and voltage of the $\mathrm{AC}$ network interface, firing angle, and commutation overlap. For each phase of an $\mathrm{AC}$ network interface to a 6-pulse bridge, the current generated is defined as [4]:

$$
I_{g}=2 \frac{\sqrt{3}}{\pi} I_{d c} \sum_{n=1}^{\infty} \frac{1}{n} \sin (n \omega t), n=6 k \pm 1, k \in \mathbb{N}
$$

Equation 1 describes the current generated on a single converter-side phase of an AC converter transformer, $I_{g}$, as a function of the DC link current $I_{d c}$ on a six-pulse converter. Twelve-pulse converter topologies can allow two transformers to be respectively configured in star-star and star-delta winding arrangements to offset one three-phase converter input with respect to the other by a $30^{\circ}$ phase shift. This is the system modelled by the simulation in Figure 1, and generates fewer harmonics as $n$ instead increases in increments of twelve, reducing total overall harmonic distortion. Harmonics on the $\mathrm{AC}$ interface are converse to those on the DC link itself, which instead adhere to the formula: $n_{d c}=6 k$ for the same values of $k$. A 12-pulse line commutated converter therefore generates $11^{\text {th }}$, $13^{\text {th }}, 23^{\text {rd }}, 25^{\text {th }}$, etc. harmonics on the source side and $12^{\text {th }}, 24^{\text {th }}$, $36^{\text {th }}$, etc. on the DC side [6]. This way, fifth and seventh harmonics can be reduced on a converter transformer's primary side, but still exist on secondary windings [6]. Figure 2 shows unfiltered characteristic harmonic distortion on one phase current for a 12-pulse converter with star-star and star-delta connected transformers at the rectifier AC interface, shown in green, and equivalently at the inverter interface, in blue.

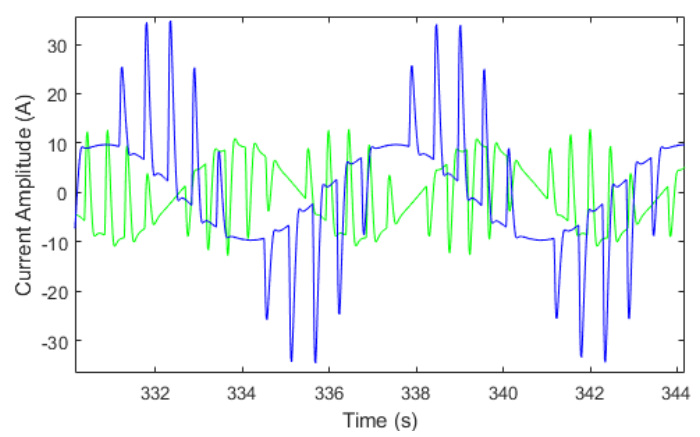

Figure 2: Simulated inverter phase current characteristic harmonics

Additional sources for high-frequency signal artefacts in HVDC converter stations might include: switching capacitor banks to compensate reactive power in line with daily load profiles; the use of static compensators or similar power electronics to meet discrepancies in requirements for reactive power support with that supplied by the filter banks switched in at any given time; imperfect commutation; and commutation overlap. AC voltage drops (due to faults or disturbances, for instance) may cause valves to experience occasional commutation failures (p321, [3]). Power factors, loading, and firing angles further affect characteristic harmonics.

\section{A. Firing Angle}

Small firing angles are preferable since nonlinear switching elements at non-zero firing angles introduce an immediate step change in the current crossing a converter bridge arm, introducing a harmonic magnitude related to the angle.

\section{B. Commutation Overlap}

In LCC systems, AC current directly commutates each arm of a converter bridge since current reversal returns a thyristor to a blocking state. Steep current transients are mitigated by inductances, so in the presence of large converter transformers, the current transitions needed for commutation are not immediate: instead, a decreasing current on one bridge arm overlaps with an increasing one on another. This distorts the DC current waveform and introduces further high frequency signal components dependent on the angle of overlap, $\gamma$. 


\section{CONVERTER StATION AsSETS}

Filtering assets attenuate certain harmonic frequencies, but also help to provide the reactive power that LCC converters consume. Hence, it is important to size such assets to meet these dual objectives in tandem, protect them from gradual deterioration and to limit the variability of operating conditions to minimise unwanted changes. Filter components are typically grouped according to the harmonic frequencies they aim to eliminate: common filter arrangements have an initial large capacitor bank to support reactive power, and a set of series connected filters each with parallel resistive, capacitive, and inductive components to select for and dissipate particular frequencies, before connecting to ground through a resistor.

\section{A. Asset Degradation}

As these assets degrade over time, their electrical properties are likely to change: any breakdown in dielectric strength between windings of inductor coils could consequently increase the stress placed on those that remain, and any short circuits between foils of capacitor elements could result in reduced overall capacitance. As a result, any reduction in filter resistances could compromise filters' ability to dissipate harmonic energy, changes in inductor value can influence the tuning of filters, and damage to capacitor modules can not only affect the frequencies at which filters are tuned, but might also reduce the reactive power support they provide. To some extent, components such as capacitor banks are designed to accommodate some failure by including a number of redundant elements. Their modular nature also facilitates replacement in the event that fault locations can be identified. Filter components are switched in and out, and their values are varied in order to demonstrate the effects of each on harmonic attenuation. For the purposes of simulating such asset degradation, it is useful to firstly understand the relationship between reactive power support and tuning for the same set of assets. Filter detuning can occur for a single-tuned band-pass filter according to a frequency deviation [7]:

$$
\delta_{e k v}=\frac{\Delta f}{f_{0}}+\frac{1}{2}\left(\frac{\Delta C}{C}+\frac{\Delta L}{L}\right)
$$

Contributing factors to frequency deviation (filter detuning) can be: manufacturer tolerances, temperature changes; failures and aging; variations of power system frequency leading to changes in the harmonic frequency [7]. Should detuning occur over an asset's lifetime, it could lead to an increase in harmonics seen on AC network interfaces and thereby increase the threat posed by a converter to other converter station, and pre-existing AC network, assets. Moreover, it can be made readily correctible if the appropriate mechanisms are in place at converter stations, and if there is the means to take a measure of any drift in capacitive and inductive values in the context of the converter operating conditions. IEC 61000-3-6:2008 specifies an $8 \%$ limit on total harmonic distortion (THD), and limits on specific AC transmission network harmonics as in Table 1, which shows maximum permitted harmonic voltages as a percentage of a fundamental voltage (in excess of $35 \mathrm{kV}$ ) for each harmonic order, provided here for convenience [8].
TABLE 1. HARMONIC LIMITS [8]

\begin{tabular}{|c|c|c|c|c|c|}
\hline \multicolumn{2}{|c|}{ Neither multiples of 2 nor 3 } & \multicolumn{2}{|c|}{ Multiples of 3 } & \multicolumn{2}{|c|}{ Multiples of 2 } \\
\cline { 1 - 1 } $\begin{array}{c}\text { Harmonic } \\
\text { Order }\end{array}$ & $\begin{array}{c}\text { Harmonic } \\
\text { Voltage (\%) }\end{array}$ & $\begin{array}{c}\text { Harmonic } \\
\text { Order }\end{array}$ & $\begin{array}{c}\text { Harmonic } \\
\text { Voltage (\%) }\end{array}$ & $\begin{array}{c}\text { Harmonic } \\
\text { Order }\end{array}$ & $\begin{array}{c}\text { Harmonic } \\
\text { Voltage (\%) }\end{array}$ \\
& $>35 \mathrm{kV}$ & & $25 \mathrm{kV}$ & & $>35 \mathrm{kV}$ \\
\hline 5 & 2 & 3 & 2 & 4 & 1.4 \\
\hline 7 & 2 & 9 & 1 & 6 & 0.8 \\
\hline 11 & 1.5 & 15 & 0.3 & 8 & 0.4 \\
\hline 13 & 1.5 & 21 & 0.2 & 0.4 \\
\hline $17 \leq \mathrm{h} \leq 49$ & $1.2 \times(17 / \mathrm{h})$ & $21<\mathrm{h} \leq 45$ & 0.2 & $10 \leq \mathrm{h} \leq 50$ & $0.19 \times(10 / \mathrm{h})+0.16$ \\
\hline
\end{tabular}

\section{Methodology}

Steady-state signals can be captured and converted to the frequency domain for analysis, and by including a block to calculate the THD at either end of the LCC link, it is possible to illustrate the effects of different operating conditions on the level of harmonics present on the interface with the $\mathrm{AC}$ network. With filters disconnected, simulations are repeated for different values of firing angle, to view the frequency domain characteristics of the resulting signals. Filters are then reconnected at different values of frequency deviation (the cause of this is abstracted and it is assumed the deviation in frequency is sufficient). Total harmonic distortion is calculated for each case: firing angles from 0.4 to 1 per unit, and for filter frequency deviations in increments of 0.5 harmonic indices around the nominal frequency tuning of each filter group. For simplicity, all changes made at one terminal are replicated at the other. Reactive power provided can then be altered in line with the change in tuned frequency to mimic a real system.

\section{RESULTS}

The firing angle $\alpha$ was initially varied between 1 and 0.4 per unit values, to illustrate the impact of changing firing angle on the harmonic content of the converter terminal current signals. However, despite firing angle changes, the maximum total harmonic distortion from these simulations remained as a constant 1.1926 and a constant 4.8632 respectively measured on a single phase current at the AC terminals of the rectifier and inverter stations. Moreover, for each of the test cases outlined in Table 2, there was minimal variation in maximum THD due to variation in firing angle. Half-harmonic values of frequency drift are introduced to different filters to give the results in Table 2, where $\mathrm{n}$ represents the harmonic magnitude. Table 2 also illustrates how different configurations of filtering assets can affect the maximum THD. Each change was made in isolation, keeping other parameters constant, so each value is relative to nominal values of maximum THD at rectifier and inverter station terminals, respectively 1.1926 and 4.8632 . Larger variations occur as filter banks are disconnected in their entirety, and frequency drift of the 24th filter tuning gives least variation. Frequency domain graphs were obtained for the phase A voltage at the interface of the rectifier with the $\mathrm{AC}$ network under different conditions, and are shown in Figure 3. 
TABLE $2 . \quad$ TEST CASES

\begin{tabular}{|l|l|c|c|}
\hline \multirow{2}{*}{$\begin{array}{c}\text { Filter Bank } \\
\text { Harmonic }\end{array}$} & \multicolumn{3}{|c|}{ Maximum THD at Converter Terminals } \\
\cline { 2 - 4 } & \multicolumn{1}{|c|}{ Change } & Rectifier & Inverter \\
\hline $11^{\text {th }} 11^{\text {th }}$ & $+0.5 n$ Frequency Deviation & 1.1945 & 4.8606 \\
\hline $13^{\text {th }}$ & $-0.5 n$ Frequency Deviation & 1.1935 & 4.8651 \\
\hline $13^{\text {th }}$ & $+0.5 n$ Frequency Deviation & 1.1917 & 4.8620 \\
\hline $24^{\text {th }}$ & $-0.5 n$ Frequency Deviation & 1.1925 & 4.8634 \\
\hline $24^{\text {th }}$ & $+0.5 n$ Frequency Deviation & 1.1927 & 4.8630 \\
\hline $11^{\text {th }}$ & Disconnected & 1.2488 & 4.9379 \\
\hline $13^{\text {th }}$ & Disconnected & 1.2448 & 4.9403 \\
\hline $24^{\text {th }}$ & Disconnected & 1.2271 & 4.9357 \\
\hline Q $^{(\text {Support })}$ & Disconnected & 1.2481 & 4.9289 \\
\hline $24^{\text {th }} \&$ Q & Disconnected & 1.3364 & 5.1000 \\
\hline
\end{tabular}
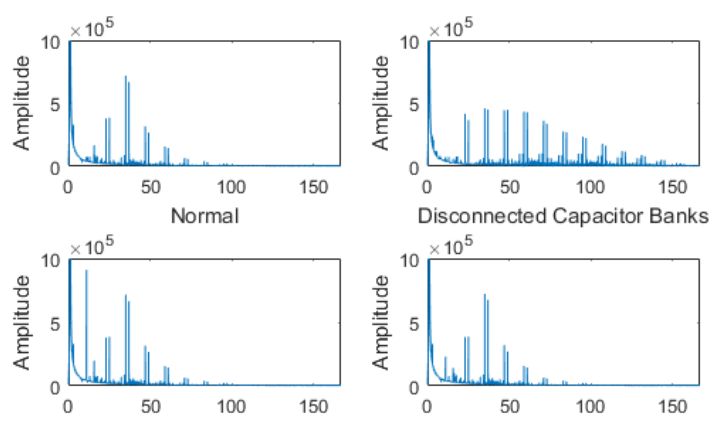

$-0.5 n$ Frequency Drift on 11 th Filters $+0.5 n$ Frequency Drift on 11th Filters
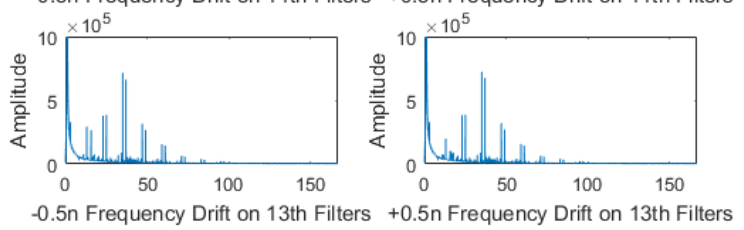

Figure 3: Rectifier interface voltage harmonics under different conditions

\section{DISCUSSION}

Using the provided example model necessitates using a firing angle measured in per unit values. Since firing angle corresponds to line loading, it is assumed that $\alpha=1 \mathrm{p} . u$. means the line is fully loaded and that $\alpha=0^{\circ}$, with decreasing values in per unit corresponding to increasing angles. Since firing and commutation overlap angles typically are small and do not induce large harmonics, this would be an acceptable approximation for typical operating scenarios, but during fault conditions these parameters can increase to higher values. Faults have not been modelled in this paper. However, the firing angle begins at a minimum of 0.4 per unit, since no representative results can be obtained below that value.
Changing the commutation overlap angle $\gamma$ is more cumbersome in this model: the rate of change of current is a function of the inductive parameters on the $\mathrm{AC}$ converter interface, which is influenced by inductances throughout the model including reactive filter components. Any deviation to the value of a filter capacitance or reactance can both affect tuning and the reactive power supplied. It has been omitted as a control parameter, but is known to contribute to harmonics.

The comparatively larger change in harmonic distortion as a result of the complete disconnection of different filter banks suggests that the reactive power contribution does more for harmonic attenuation on LCC interfaces than the specific tuned frequencies of the filters, which is corroborated by the increase in THD resulting from the combined disconnection of the capacitor banks in place to provide reactive power support. The smaller changes resulting from frequency drift of the filter banks double-tuned around the $24^{\text {th }}$ harmonic indicate the lesser magnitudes of harmonics at these frequencies relative to those of the $11^{\text {th }}$ and $13^{\text {th }}$ characteristic harmonics.

Any filtering system would ideally be configured to avoid resonances, with consideration of how transmission networks might gradually change toward a reduction in synchronous generation, an increased number of connected interconnection, and a greater number of converter-connected generation all of which lead toward potential changes in the resonant frequency. Transmission systems are changing with the introduction of HVDC systems (particularly subsea connections), changes to synchronous generation, and the connection of new generation via converters; and the resonant characteristics of transmission infrastructure is changing too. HVDC converter stations offer opportunity for increased control, which could be used to mitigate resonant oscillations in $\mathrm{AC}$ transmission networks, but only if suppliers and operators collaborate as a prerequisite [9].

The diagrams in Figure 3 are scaled similarly for easy comparison, and at first glance show that the greatest harmonic increases result from the reduced reactive power support available as capacitor banks are disconnected. The influence of local reactance influences voltage harmonics via the overlap angle $\gamma$, and also readily affects values of THD provided in Table 2 which are based on current. In the case where the $11^{\text {th }}$ harmonic filter is detuned by $-0.5 n$, there is a clear increase in magnitude of the $11^{\text {th }}$ harmonic, in addition to variation in the other components. As the filter tuning for the $11^{\text {th }}$ harmonic drifts to higher frequencies, or as that of the $13^{\text {th }}$ decreases in frequency, the magnitudes of these specific harmonics might increase, but each also has potential to attenuate the other with sufficient change in frequency. THD is an established metric for assessing overall signal quality, but since it provides a single measure for a number of underlying phenomena, it could mask such effects. Spectral leakage in the diagrams shown in Figure 3 reduces their clarity at small values, however, but inverter harmonics captured at the rectifier can also be seen in these frequency spectra.

As shown in Figure 3, harmonics remain on the system despite filtering. Such signals can inevitably seem inconsequential in comparison to the magnitude of the fundamental waveform, but these signals can still reach significant magnitudes in absolute terms. The basis of this 
study is just an example, but the attenuated harmonics can still reach magnitudes of around $50 \mathrm{kV}$. These represent repeated step changes in voltages and currents passed by a converter. Harmonics reduce the quality of a power supply, and can damage existing electrical assets. To some extent, parameters of the network can further help to mitigate harmonic currents, but since LCC converter stations consume reactive power, they are sometimes sited near to synchronous generation to protect against reactive power consumption. This might suggest assets associated with synchronous generators - those already critical due to a strategic position - could be at risk of degradation from high frequency electrical signals they had hitherto not experienced throughout their operational life.

\section{A. Further Work}

Characteristic parameters for each filter block have been specified, but being able to convert these to straightforward values of resistance, inductance and capacitance would allow more representative changes to be made and simulated to evaluate changes as a result of degradation of each asset type. This could be achieved by specifying each block according to its design characteristics (as used currently), but then testing it at its filtering frequency to obtain a resistance, and then again at the fundamental frequency to evaluate the reactance which can be verified relative to the resistance using a quality factor.

No particular scheme has formed the basis of this indicative study, however, and values chosen do not represent those of a specific system. There is a need for modelling and simulation capabilities for the more detailed aspects of power electronics. Current options include the ability to simulate power electronic components in Simulink, but with limited control over their operation, and some auxiliary components are omitted from detailed thyristor models. For instance, Simulink's unified switch omits a way to model errors. Open source options exist [10] [11], but are less accessible, and conventional SPICE packages appear less well suited to high capacity power electronic applications as they are to low-power circuitry.

\section{VIII.CONCLUSIONS}

This paper introduces the type of signals which can be generated by a converter station, via simulation. Such signals are shown to vary in accordance with operating condition on an HVDC link, and to be for the most part attenuated by AC filter components typically installed for such schemes. In particular, variation in load, AC system power factor, and firing angle all contribute to the severity of harmonics generated by a converter, suggesting that HVDC control systems ought to be cognisant of harmonics generated as a consequence of control strategies. Filtering assets are also simulated to indicate their influence on these unwanted harmonics.

\section{REFERENCES}

[1] M. Hotz, W. Utschick, "A hybrid transmission grid architecture enabling efficient optimal power flow", IEEE Transactions on Power Systems, Vol. 31, No. 6, 2016

[2] CIGRE, WG C4.49 "Proposal for the creation of a new working group: multi-frequency stability of converter-based modern power systems".

[3] B. M. Weedy, B. J. Cory, N. Jenkins, J. B. Ekanayake, G. Strbac, Electric Power Systems. John Wiley \& Sons Ltd., Fifth edition, 2012.

[4] D. Jovcic, K. Ahmed, High Voltage Direct Current Transmission: Converters, Systems, and DC Grids. John Wiley \& Sons Ltd., 2015.

[5] "Thyristor-based HVDC transmission system", [Online]. Accessed at: https://uk.mathworks.com/help/physmod/sps/examples/thyristor-basedhvdc-transmission-system-detailed-model.html

[6] G. Bhuvaneswari and B. C. Mahanta, "Analysis of converter transformer failure in HVDC systems and possible solutions", IEEE Transactions on Power Delivery, Vol. 24, No. 2, April 2009.

[7] Alstom Grid Ltd., HVDC: Connecting to the Future.

[8] International Electrotechnical Commission, Electromagnetic compatibility (EMC) - Part 3-6: Limits. 61000-3-6, 2008.

[9] P. Mohapatra, "Innovations of the transmission network" [Lecture]. IET Teacher Building, Glasgow, March 2018.

[10] S. Iyer, "Python power electronics" [Online]. Accessed at: http://pythonpowerelectronics.com

[11] "Simulate electronic circuit using python and the ngspice / xyce simulators" [Online]. Accessed at: https://pyspice.fabrice-salvaire.fr 